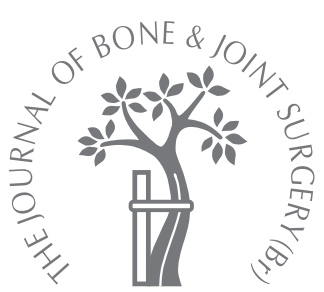

V. Khanduja, H. S. Somayaji, P. Harnett, M. Utukuri, G. S. E. Dowd

From the Royal Free Hospital and the Wellington Knee Unit, London, England

In. Khanduja, MRCS(G), MSc, FRCS(Tr \& Orth), Knee Fellow Royal Free Hospital and The Wellington Knee Unit, Wellington Place, St John's Wood, London NW8 9LE, UK.

= H. S. Somayaji, MS, MRCS, Specialist Registrar Department of Trauma \& Orthopaedics

Watford General Hospital Watford, Hertfordshire WD18 OHB, UK.

I. P. Harnett, BSc, MBChB, MRCS, Senior House Officer M. Utukuri, MS, FRCS(Tr \& Orth), Senior Clinical Fellow a G. S. E. Dowd, MD, MCh Orth, FRCS, Consultant Orthopaedic Surgeon Department of Orthopaedics Royal Free Hospital, Pond Street, London NW3 20G, UK.

Correspondence should be sent to Mr V. Khanduja at 109a, Queen's Avenue, Watford, Hertfordshire WD18 7NU, UK e-mail:

vikaskhanduja@aol.com

(C2006 British Editorial Society of Bone and Joint Surgery doi:10.1302/0301-620X.88B9. $17591 \$ 2.00$

$J$ Bone Joint Surg $[\mathrm{Br}]$ 2006;88-B:1169-72.

Received 30 December 2005; Accepted after revision 10 May 2006

\title{
Combined reconstruction of chronic posterior cruciate ligament and posterolateral corner deficiency
}

\author{
A TWO- TO NINE-YEAR FOLLOW-UP STUDY
}

We report a retrospective analysis of the results of combined arthroscopically-assisted posterior cruciate ligament reconstruction and open reconstruction of the posterolateral corner in 19 patients with chronic (three or more months) symptomatic instability and pain in the knee.

All the operations were performed between 1996 and 2003 and all the patients were assessed pre- and post-operatively by physical examination and by applying three different ligament rating scores. All also had weight-bearing radiographs, MR scans and an examination under anaesthesia and arthroscopy pre-operatively. The posterior cruciate ligament reconstruction was performed using an arthroscopically-assisted single anterolateral bundle technique and the posterolateral corner structures were reconstructed using an open Larson type of tenodesis.

The mean follow up was 66.8 months (24 to 110). Pre-operatively, all the patients had a grade III posterior sag according to Clancy and demonstrated more than $20^{\circ}$ of external rotation compared with the opposite normal knee on the Dial test. Post-operatively, seven patients $(37 \%)$ had no residual posterior sag, $11(58 \%)$ had a grade I posterior sag and one $(5 \%)$ had a grade II posterior sag. In five patients $(26 \%)$ there was persistent minimal posterolateral laxity. The Lysholm score improved from a mean of 41.2 (28 to 53 ) to 76.5 (57 to 100$)(p=0.0001)$ and the Tegner score from a mean of 2.6 (1 to 4$)$ to $6.4(4$ to 9$)(p=$ 0.0001).

We conclude that while a combined reconstruction of chronic posterior cruciate ligament and posterolateral corner instability improves the function of the knee, it does not restore complete stability.

A combined injury of the posterior cruciate ligament (PCL) and the posterolateral corner results in a complex pattern of instability with significant functional limitation. ${ }^{1,2}$ These injuries are rare and difficult to diagnose at initial presentation, which may explain their frequent presentation as a chronic condition. The rarity of this injury, coupled with the complexity of reconstruction and variable results, has produced few reports on the subject. ${ }^{2-5}$

In an attempt to increase the understanding of the subject, we present our results of combined reconstruction of the PCL and the posterolateral corner for chronic instability and pain of three or more months in duration, with a two- to nine-year follow-up.

\section{Patients and Methods}

From our database we identified 19 patients who underwent a combined reconstruction of the PCL and posterolateral corner for chronic symptomatic instability and pain. None required a valgus tibial osteotomy for a varus thrust before reconstruction. Five of the 19 patients had undergone previous arthroscopies elsewhere for meniscal tears (four medial and one lateral).

There were 18 men and one woman in the study group. The mean age of the patients at the time of surgery was 29.6 years ( 21 to 47 ) with a mean interval from injury to surgery of 27.3 months (3 to 105). All 19 patients were available for follow-up at a mean of 66.8 months (24 to 110). The mechanism of injury was contact sports, including football or rugby in 11 patients, road-traffic accidents in four and non-contact sport including skiing or trampolining in four.

All the patients were assessed by the senior author (GSED) pre- and post-operatively by physical examination and using three different ligament rating scores. In addition, all had a weight-bearing full-length radiograph and, where necessary, stress radiographs. MR scans and an examination under anaesthesia with 
arthroscopy were also performed on all the patients preoperatively. The posterior sag and posterior drawer tests were used to assess laxity of the PCL. ${ }^{6}$ Posterolateral corner structures were assessed using the $\mathrm{Dial}^{7}$ test, the varus stress test and the reverse pivot shift test with the opposite normal knee being used as a control.

The posterior drawer test was graded using Clancy's classification. ${ }^{8}$ In grade 1 injuries, at $90^{\circ}$ of knee flexion, the anterior tibial crest remains anterior to the femoral condyles, but has sagged when compared with the opposite, normal knee. In grade 2 injuries the tibial crest is flush with the femoral condyles and in grade 3 the tibial crest lies posterior to the femoral condyles. The Dial test was performed at $30^{\circ}$ and $90^{\circ}$ of knee flexion in the prone position. Increased external rotation at $30^{\circ}$ knee flexion, but not at $90^{\circ}$, indicated an isolated injury to the posterolateral corner, whereas increased external rotation at both angles suggested an injury to both PCL and posterolateral corner. ${ }^{7}$ The reverse pivot shift test was considered positive if there was a feeling of reduction when the flexed, externallyrotated knee was extended with a valgus stress. ${ }^{9}$

All the operations were performed by the senior author (GSED). The PCL was first reconstructed using an arthroscopically-assisted single anterolateral bundle technique. ${ }^{10}$ In three patients hamstring and patellar tendon autografts were used for the reconstruction. In the remaining 16 patients, fresh-frozen tendo Achillis allograft was used for the reconstruction. All the grafts were fixed with the aid of soft-tissue interference screws. The posterolateral structures were then reconstructed using a Larson type of tenodesis. $^{11}$

Patients followed a strict rehabilitation protocol after surgery. They remained full weight-bearing in a knee brace which was locked in full extension for the first three weeks. Subsequently, they were allowed to commence knee flexion up to $30^{\circ}$ within the brace. At six weeks, after the brace had been discarded, further flexion was permitted and, once the patient had gained good control of their leg, closed chain kinetic exercises were encouraged. Return to sports was not permitted for up to nine months from surgery.

All the patients were followed up at regular intervals by an independent surgeon. The final examination was performed by trainees (HSS and $\mathrm{PH}$ ) in all the patients. Apart from objective clinical examination, a subjective assessment of function was made using the Tegner and Lysholm ${ }^{12}$ activity scale and scoring system and the International Knee Documentation Committee (IKDC) scale. ${ }^{13}$

Statistical analysis was performed using SPSS version 11 (SPSS Inc., Chicago, Illinois). A p value was calculated using the Wilcoxon signed rank test and Mann-Whitney U test as appropriate. A p value of less than 0.05 was considered significant.

\section{Results}

At the pre-operative arthroscopy, none of the patients was found to have a meniscal tear. However, of the four patients
Table I. Subjective assessment using the Tegner and Lysholm ${ }^{12}$ score

\begin{tabular}{lcc}
\hline Scoring system & Mean (range) & p value \\
\hline Pre-operative Tegner & 2.6 (1 to 4$)$ & \\
Post-operative Tegner & 6.4 (4 to 9$)$ & 0.0001 \\
Pre-operative Lysholm & $41.2(28$ to 53$)$ & \\
Post-operative Lysholm & 76.5 (57 to 100$)$ & 0.0001 \\
\hline
\end{tabular}

Table II. The International Knee Documentation Committee (IKDC) grades $^{13}$

\begin{tabular}{lcc}
\hline IKDC grade & Pre-operatively & Post-operatively \\
\hline A & 0 & 6 \\
B & 0 & 11 \\
C & 0 & 2 \\
D & 19 & 0 \\
\hline
\end{tabular}

who had previously undergone medial meniscectomy elsewhere, all had grade I osteoarthritic changes ${ }^{14}$ of the articular cartilage and another two had grade II changes in the medial compartment.

Physical examination. All 19 patients had a grade III posterior drawer and a positive reverse pivot shift test pre-operatively. In addition, all had more than $20^{\circ}$ of external rotation of the tibia compared with the opposite normal knee on the Dial test. At the final post-operative follow-up, seven patients $(37 \%)$ had no posterior sag (grade 0), 11 $(58 \%)$ had a grade I posterior sag and only one $(5 \%)$ had a grade II posterior sag. In 14 patients $(74 \%)$, the Dial test and varus test were negative. In the remaining five patients $(26 \%)$ there was residual minimal posterolateral laxity.

Subjective assessment. The mean pre- and post-operative Tegner and Lysholm scores are shown in Table I. The IKDC scores are shown in Table II. The Lysholm and Tegner scores improved consistently for both the allograft and the autograft groups. All the patients felt that they were improved by the procedure and were satisfied with the outcome. However, six patients (32\%) continued to experience minimal instability. Only eight patients $(42 \%)$ had a Lysholm score above 80 and $13(68 \%)$ were able to return to their pre-injury level of activity. Of the six patients with arthroscopically-identified articular cartilage changes with associated pain, the four with grade I changes had a reduction in pain from moderate to mild but the remaining two with grade II changes continued to experience moderate pain. In addition, two other patients complained of mild pain, making a total of eight $(42 \%)$.

Seven patients $(37 \%)$ required a further procedure to remove the tibial fixation screw as it was uncomfortable. Two patients developed a superficial infection of the posterolateral corner wound because of friction from the brace, which healed following oral antibiotics and adjustment of the brace. Two patients required a manipulation under anaesthesia at six weeks following surgery because of post-operative stiffness, and improved subsequently. There were no neurovascular or allograft-related complications. 


\section{Discussion}

One of the first reports of PCL reconstruction was published in 1917 by Hey-Groves. ${ }^{15}$ Since then there has been a sporadic interest in reconstruction of the PCL with little reported success. However, in the past decade there has been an increased understanding of the biomechanics and function of the PCL and posterolateral corner, the physical signs associated with disruption of these structures and an improvement in the investigation of these injuries. New techniques of reconstruction have been developed, but reports of the results remain limited. ${ }^{2-5,16}$

The incidence of injury to the PCL in all acute injuries to the knee varies between $2 \%$ during sport and $40 \%$ following road traffic accidents. ${ }^{17}$ Of these, between $50 \%$ and $90 \%$ involve associated injury to the other structures in the knee with the posterolateral corner being the most frequent. ${ }^{17}$ In a report by Fanelli and Edson ${ }^{17}$ only $1.4 \%$ of patients with a haemarthrosis had an isolated injury of the PCL, while rupture of the PCL with injury to the posterolateral corner occurred in $16 \%$.

The biomechanical function of the PCL is the primary restraint of posterior translation of the tibia on the femur. It also acts as a secondary restraint to external rotation of the tibia and varus angulation. ${ }^{18}$ The posterolateral corner has been anatomically defined as a structure divided into three layers, from superficial to deep. ${ }^{19}$ The most superficial layer consists of the iliotibial tract and biceps femoris with its posterior expansion. The second layer consists of the lateral collateral ligament, the quadriceps retinaculum and the patellofemoral ligaments. The deep layer consists of the popliteus tendon, the arcuate ligament and the popliteofibular ligament. Biomechanically, the posterolateral corner restricts excess external rotation of the lateral condyle of the tibia and resists varus angulation with an additional dynamic element through the popliteus muscle and tendon. ${ }^{4,19}$ Therefore, it can be understood that a combined injury to the PCL and posterolateral corner produces a much more profound effect on the biomechanics of the joint than an isolated injury to the PCL.

The common mechanism which leads to this complex injury is a blow to the inner aspect of the thigh producing a varus deformity to the extended or almost extended knee. ${ }^{3,18}$ This mechanism is often the result of a tackle in football or rugby as was seen in our series. External rotation injuries in skiers may also produce this pattern of injury. The presence or absence of a posterior sag is the essence of clinical examination for a ruptured PCL. ${ }^{6}$ As far as the posterolateral corner is concerned, the Dial test for tibial external rotation is the most sensitive. In the case of chronic rupture of both these structures there is usually grade II or greater posterior sag with a positive Dial test. ${ }^{20}$ Examination of gait is essential as some of these patients may walk with a varus thrust or a slightly flexed knee and an internally rotated foot to prevent painful posterior subluxation of the lateral tibial plateau. ${ }^{4,21}$ Although we performed KT 1000 arthrometer measurements routinely for all patients with ligament injuries, we did not find them useful in assessing this combined injury.

Routine radiographs of the knee were obtained to detect osteoarthritic changes associated with chronic PCL insufficiency and a long-leg radiograph to evaluate the possible need for a re-alignment osteotomy for excessive varus before the ligament reconstruction. ${ }^{22}$ None of our 19 patients required a re-alignment osteotomy. Stress radiographs may be used to evaluate PCL and posterolateral corner deficiency. ${ }^{18}$ However, in our experience they are difficult to interpret and their use has not been validated in combined PCL and posterolateral corner injuries. MR scans are only used as an adjunct to clinical diagnosis and are obtained to determine the location of the tear, damage to the articular cartilage and any other ligament injuries. However, it has been shown that an MR scan may fail to show a PCL tear in chronic cases because of the ability of PCL to heal, albeit in an elongated form. ${ }^{23}$

The results of reconstruction of the PCL have, until recently, been generally less satisfying than those for the anterior cruciate ligament. ${ }^{24}$ The reasons are multifactorial, including difficulty in accurate positioning of the graft, delay in diagnosis before the onset of degenerative change and also the lack of awareness of other instabilities, especially those of the posterolateral corner, which require treatment. ${ }^{18,20}$ The chronic laxity of the posterolateral corner must be addressed using some form of tenodesis incorporated in the surgical treatment plan to avoid increased strain on the reconstructed PCL graft, which may otherwise become lengthened and lax. ${ }^{25}$

Our results compare favourably with those reported by Wang ${ }^{5}$ and Noyes and Barber-Westin. ${ }^{26}$ However, compared with Fanelli and Edson, ${ }^{3}$ who have the largest reported series of PCL and posterolateral corner reconstructions, our results are inferior. We were able to restore a normal tibial step-off and a normal posterior drawer in only $37 \%$ of the patients compared with $70 \%$ reported by Fanelli and Edson. ${ }^{3}$ In addition, only $74 \%$ of our patients had no residual posterolateral laxity compared with $98 \%$ in their series. However, they performed a biceps tenodesis as described by Clancy ${ }^{8}$ for posterolateral corner laxity. The disadvantages of the procedure include over-tightening of the lateral structures leading to the potential risk of degenerative change, which they observed in $71 \%$ of their patients. Additionally, if the tenodesis is too tight the biceps can separate from the fixation screw. Finally, a biceps tenodesis results in the loss of the only lateral knee flexor.

Although, all of our patients had a functionally stable knee, only $13(68 \%)$ could return to their pre-injury activity status compared with $100 \%$ in Fanelli and Edson's series. $^{3}$ Six $(32 \%)$ continued to have minimal subjective instability and eight $(42 \%)$ continued to be in some pain.

Harvesting autograft increases operative time and is associated with donor-site morbidity. The advantages of allograft include decreased surgical time, improved cosmesis, reduced post-operative pain and reduced complica- 
tions. ${ }^{18,27}$ However, concern exists that using an allograft may increase the risks of disease transmission, delayed healing and immunological reactions and be subject to limited availability. ${ }^{27,28}$ In our experience, with a comprehensive pre-operative evaluation, a carefully-executed surgical procedure, and supervised programme of rehabilitation, satisfactory results can be obtained with either of the graft choices. Other studies have also shown little difference in results between the two graft sources. 2,20

Finally, our results show that although there is functional improvement after reconstruction of the PCL and posterolateral corner, there may only be a modest improvement in objectively-assessed knee stability. This might be because of difficulty in identifying the best site for placement of the femoral tunnel and because the procedures described for reconstruction of the posterolateral corner are static in nature whereas the popliteus complex is a dynamic structure and may also have a proprioceptive role in balancing the lateral compartment of the knee. Unless these problems are addressed in a dynamic form, results of such combined reconstructions will provide an imperfect solution.

No benefits in any form have been received or will be received from a commercial party related directly or indirectly to the subject of this article.

\section{References}

1. Sekiya JK, Haemmerle MJ, Stabile KJ, Vogrin TM, Harner CD. Biomechanical analysis of a combined double-bundle posterior cruciate ligament and posterolateral corner reconstruction. Am J Sports Med 2005:33:360-9.

2. Fanelli GC, Giannotti BF, Edson CJ. Arthroscopically assisted combined posterior cruciate ligament/posterior lateral complex reconstruction. Arthroscopy 1996;12: 521-30.

3. Fanelli GC, Edson CJ. Combined posterior cruciate ligament-posterolateral reconstructions with Achilles tendon allograft and biceps femoris tendon tenodesis: 2- to 10-year follow-up. Arthroscopy 2004;20:339-45.

4. Mariani PP, Becker R, Rihn J, Margheritini F. Surgical treatment of posterior cruciate ligament and posterolateral corner injuries: an anatomical, biomechanical and clinical review. Knee 2003:10:311-24.

5. Wang CJ. Injuries to the posterior cruciate ligament and posterolateral instabilities of the knee. Chang Gung Med J 2002;25:288-97.

6. Rubenstein RA Jr, Shelbourne KD, McCarroll JR, VanMeter CD, Rettig AC. The accuracy of the clinical examinations in the setting of the posterior cruciate ligament injuries. Am J Sports Med 1994;22:550-7.

7. Veltri DM, Warren RF. Operative treatment of posterolateral instability of the knee. Clin Sports Med 1994;13:615-27.
8. Bisson LJ, Clancy WG. Isolated posterior cruciate ligament injury and posterolateral laxity. In: Chapman M, ed. Chapman's orthopaedic surgery. Philadelphia: JB Lippincott, 2001:2393-416.

9. Jacob RP, Staubli HU, Deland JT. Grading the pivot shift: objective tests with implications for treatment. J Bone Joint Surg [Br] 1987;69-B:294-9.

10. Dowd G, Duri ZA. Arthroscopically assisted posterior cruciate ligament reconstruction. In: Hunt D, ed. Minimal access orthopaedics. Radcliffe Medical Press, 1995:39-51.

11. Larson RV, Sidles JA, Beales TC. Isometry of the lateral collateral, popliteofibular ligaments and a technique for reconstruction. University of Washington Orthopaedic Research Report 1996:42-4.

12. Tegner Y, Lysholm J. Rating systems in the evaluation of knee ligament injuries. Clin Orthop 1985;198:43-9.

13. International Knee Documentation Commitee. Knee ligament injury and reconstruction evaluation. In: Aichroth PM, Cannon WD Jr, eds. Knee surgery: current practice. New York: Raven 1992:759-60.

14. Outerbridge RE. The etiology of chondromalacia patellae. J Bone Joint Surg [Br] 1961;43-B:752-7.

15. Hey-Groves EW. Operation for repair of the crucial ligaments. Lancet 1917;2:674-5.

16. Cooper DE, Stewart D. Posterior cruciate ligament reconstruction using single-bundle patella tendon graft with tibial inlay fixation: 2- to 10-year follow-up. Am J Sports Med 2004;32:346-60.

17. Fanelli GC, Edson CJ. Posterior cruciate ligament injuries in trauma patients: part II. Arthroscopy 1995; 11:526-9.

18. Dowd GS. Reconstruction of the posterior cruciate ligament: indications and results. J Bone Joint Surg [Br] 2004;86-B:480-91.

19. Veltri DM, Warren RF. Anatomy, biomechanics, and physical findings in posterolateral knee instability. Clin Sports Med 1994;13:599-614.

20. Freeman RT, Duri ZA, Dowd GS. Combined chronic posterior cruciate and posterolateral corner ligamentous injuries: a comparison of posterior cruciate ligament reconstruction with and without reconstruction of the posterolateral corner. Knee 2002:9:309-12.

21. Davies H, Unwin A, Aichroth P. The posterolateral corner of the knee: anatomy, biomechanics and management of injuries. Injury 2004;35:68-75.

22. Malone AA, Dowd GS, Saifuddin A. Injuries of the posterior cruciate ligament and posterolateral corner of the knee. Injury 2006;37:485-501.

23. Shelbourne KD, Jennings RW, Vahey TN. Magnetic resonance imaging of posterior cruciate ligament injuries: assessment of healing. Am J Knee Surg 1999;12: 209-13.

24. Jakob RP, Edwards JC. Posterior cruciate ligament reconstruction: anterior-posterior two stage technique. Sports Med Arthrosc Rev 1994;2:137-45.

25. Vogrin TM, Hoher J, Aroen A, Woo SL, Harner CD. Effects of sectioning the posterolateral structured on knee kinematics and in situ forces in the posterior cruciate ligament. Knee Surg Sports Traumatol Arthrosc 2000;8:93-8.

26. Noyes FR, Barber-Westin SD. Surgical restoration to treat chronic deficiency of the posterolateral complex and cruciate ligaments of the knee joint. Am J Sports Med 1996;24:415-26.

27. McGuire DA. Should allografts be used for routine anterior cruciate ligament reconstructions?: yes, allografts should be used in routine ACL reconstruction. Arthroscopy 2003;19:421-4

28. Lee MC, Park YK, Lee SH, Jo H, Seong SC. Posterolateral reconstruction using split Achilles tendon allograft. Arthroscopy 2003;19:1043-9. 